\title{
A Miniaturized Hemoretractometer for Blood Clot Retraction Testing
}

\author{
Zida Li, Xiang Li, Brendan McCracken, Yue Shao, Kevin Ward, and Jianping Fu*
}

Blood coagulation is a critical hemostatic process that must be properly regulated to maintain a delicate balance between bleeding and clotting. Disorders of blood coagulation can expose patients to the risk of either bleeding disorders or thrombotic diseases. Coagulation diagnostics using whole blood is very promising for assessing the complexity of the coagulation system and for global measurements of hemostasis. Despite the clinic values that existing whole blood coagulation tests have demonstrated, these systems have significant limitations that diminish their potential for point-of-care applications. Here, recent advancements in device miniaturization using functional soft materials are leveraged to develop a miniaturized clot retraction force assay device termed mHemoRetractoMeter (mHRM). The mHRM is capable of precise measurements of dynamic clot retraction forces in real time using minute amounts of whole blood. To further demonstrate the clinical utility of the mHRM, systematic studies are conducted using the mHRM to examine the effects of assay temperature, treatments of clotting agents, and pro- and anti-coagulant drugs on clot retraction force developments of whole blood samples. The mHRM's low fabrication cost, small size, and consumption of only minute amounts of blood samples make the technology promising as a point-of-care tool for future coagulation monitoring.

Z. Li, X. Li, Y. Shao, Prof. J. Fu

Department of Mechanical Engineering

University of Michigan

Ann Arbor, MI 48109, USA

E-mail: jpfu@umich.edu

B. McCracken, Prof. K. Ward

Department of Emergency Medicine

University of Michigan

Ann Arbor, MI 48109, USA

B. McCracken, Prof. K. Ward, Prof. J. Fu

Michigan Center for Integrative Research in Critical Care

University of Michigan

Ann Arbor, MI 48109, USA

Prof. J. Fu

Department of Biomedical Engineering

Department of Cell and Developmental Biology

University of Michigan

Ann Arbor, MI 48109, USA

DOI: $10.1002 /$ smll.201600274

\section{Introduction}

Blood coagulation is a critical hemostatic process involving an intricate interplay between cellular (red blood cells, white blood cells, and platelets) and acellular components (plasma proteins) of the blood. ${ }^{[1,2]}$ It is normally initiated when the blood vessel is injured and the connective tissues (particularly collagen) underlying the endothelial lining of the vessel are exposed to blood flow, leading to platelet adhesion to connective tissues, activation, and aggregation, forming a plug at the site of vessel injury. ${ }^{[3]}$ In the meanwhile, vessel injury also exposes tissue factors in connective tissues to different coagulation factors in the blood plasma, ultimately leading to a coagulation cascade which involves the conversion of fibrinogen to fibrin which then polymerizes. Importantly, activated platelets bind and exert a contractile force on fibrin strands and promote the formation of a highly crosslinked fibrin network, which further strengthens the structural integrity of 
platelet plug, leading to increased clot strength and its elastic modulus. ${ }^{[4]}$ The mechanical characteristics of the clot affect numerous aspects of hemostasis and thrombosis. ${ }^{[5]}$

Blood coagulation must be properly regulated to maintain the delicate balance between bleeding and clotting. Disorders of blood coagulation can expose patients to the risk of either bleeding disorder or thrombotic diseases such as heart attack, stroke, deep venous thrombosis, pulmonary embolism, etc. ${ }^{[6]}$ Therefore, the ability to rapidly determine the hemostatic profile of a patient is of great importance for providing timely and appropriate therapy in clinical settings such as perioperative hemostatic monitoring, ${ }^{[7]}$ transfusion therapies, ${ }^{[8]}$ thrombotic and hemophilic disease treatments, and treatments for multisystem trauma, sepsis, traumatic brain injury, and other acute diseases. ${ }^{[1]}$

Coagulation diagnostics using whole blood samples has been recently proposed as an effective method for assessing the complexity of the coagulation system and for global measurements of hemostasis. Compared to routine coagulation tests in the clinic (such as prothrombin time or PT, activated partial thromboplastin time or aPTT, and platelet count), whole-blood coagulation tests offer significant advantages of measuring the whole clotting process, starting with fibrin formation and continuing through to clot retraction and fibrinolysis, and allowing the plasmatic coagulation system to interact with blood cells including platelets, thereby providing useful additional information on platelet function. Among different whole blood coagulation tests, viscoelastic hemostasis assays including thromboelastography (TEG) and rotational thromboelastometry (ROTEM) have started to gain significant attention in the clinic. ${ }^{[9]}$ In principle, TEG and ROTEM measures the changes in the shear elastic modulus of whole blood upon clotting. For example, TEG consists of a rotating cup and a pin which is connected to a rotation sensor. Blood sample is held in the cup and the pin is submerged in the blood sample during the assay. When the blood starts clotting, its viscoelasticity changes, leading the pin to rotate along with cup. The rotational amplitude of the pin is recorded by the rotation sensor and reported for analysis of the viscoelasticity change of the blood sample. Such viscoelastic hemostasis assays have been shown to provide critical information on the interactivity of the major phases of clot formation and lysis and on the functional differences between different cellular and acellular components of the blood for differentiating the mechanism(s) related to clotting abnormalities. $^{[10]}$

Besides viscoelastic hemostasis assays, whole-blood clot retraction assays using devices such as Hemodyne Hemostasis Analysis System (HAS, Hemodyne, Inc.) are another method reported to provide important information on blood coagulation function, reporting on clot elastic modulus and platelet contractile force (or clot retraction force). ${ }^{[11,12]}$ In the HAS, whole blood samples are confined between two plates (either flat-flat or cone-cone surfaces), with the top plate attached to a strain-gage transducer. Clotting of blood results in a downward force pulling the top plate (or the clot retraction force), which is directly measured in real time by the strain-gage transducer. Using the HAS, clot retraction force has been shown to be very sensitive to platelet number and metabolic status, glycoprotein IIb/IIIa status, and the presence of antithrombin activities. ${ }^{[11]}$ Importantly, clinical applications of the HAS have demonstrated promising applications of such whole blood clot retraction assays for rapid assessment of global hemostasis ${ }^{[13]}$ and the response to a variety of procoagulant and anticoagulant medications. ${ }^{[14]}$

Despite the clinical values that the existing whole blood coagulation tests (including TEG, ROTEM, and HAS) have demonstrated through initial applications, such systems still have significant limitations in terms of costly expense to make and repair, limited sample throughput, large footprint, excessive sample size $(>0.3 \mathrm{~mL})$ that can be problematic for repetitive assays on pediatric patients, inter-assay variability, and significant user interventions, rendering them suboptimal for point-of-care applications. To miniaturize whole blood assays leveraging available microengineering technologies, many new device designs have recently been reported, such as those using capillary flow, ${ }^{[15]}$ micropost arrays, ${ }^{[16]}$ cantilever oscillation, ${ }^{[17]}$ etc. However, these miniaturized whole blood assays have only focused on measurements of blood viscoelastic properties and their correlations with blood coagulation. To account for both mechanical rigidity of fibrin network and platelet contraction, and to develop suitable point-of-care coagulation devices that are simple, inexpensive, quick, high throughput, and can be widely available, in this work we leveraged recent advancements in small scale device fabrication using functional soft materials ${ }^{[18]}$ and developed a miniaturized clot retraction assay device termed mHemoRetractoMeter (mHRM). In the mHRM, two doubly clamped mechanical strain sensing beams (in millimeter size range) made with soft elastomer polydimethylsiloxane (PDMS) were utilized for holding prescribed minute amounts of blood samples while simultaneously measuring clot retraction force during blood clotting. Kinetic curves of clot retraction force obtained by the mHRM were utilized for analysis of different major phases of clot formation and extraction of key blood clotting parameters. Using mHRM, we further conducted whole blood coagulation assays to determine the effect of assay temperature, treatments of clotting agent, and pro- and anti-coagulant on clot retraction force development.

\section{Results and Discussion}

\section{1. mHRM Device Design and Characterization}

Design of the mHRM device incorporated two doubly clamped PDMS beams with protrusions at the beam centers to hold blood samples (Figure 1a,b). The doubly clamped PDMS beams served simultaneously as mechanical strain sensors to report clot retraction forces in real time during blood clotting, through direct measurements of positions of fiducial markers engraved onto the protrusions of PDMS strain sensing beams. A fabrication protocol was established to generate the mHRM device using replica molding of PDMS (see Experimental Section; Figure S1, Supporting Information), resulting in consistent and precise geometries 
(a)
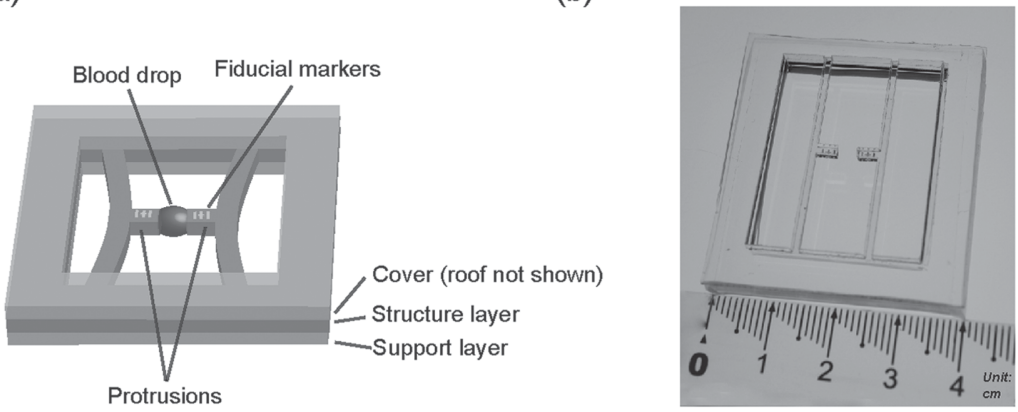

(d)

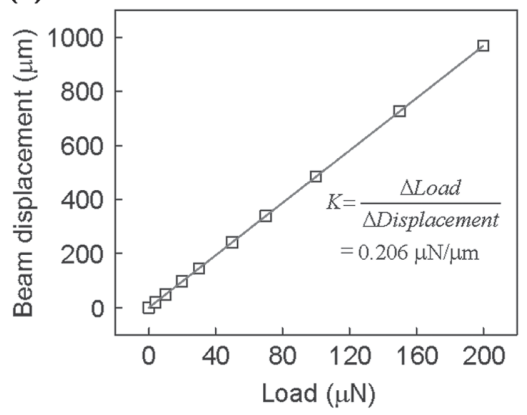

(g)

(e)

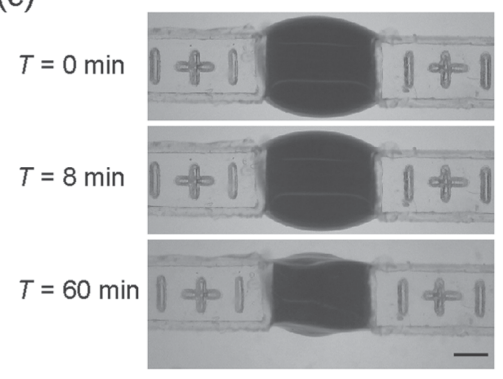

(c)

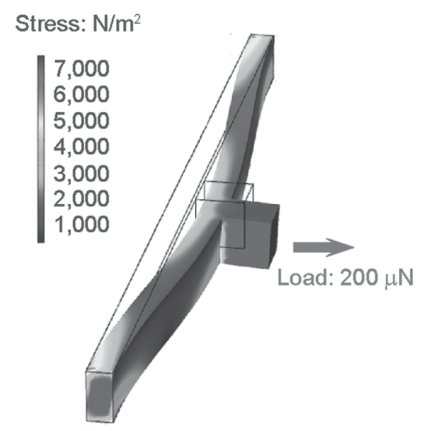

(f)

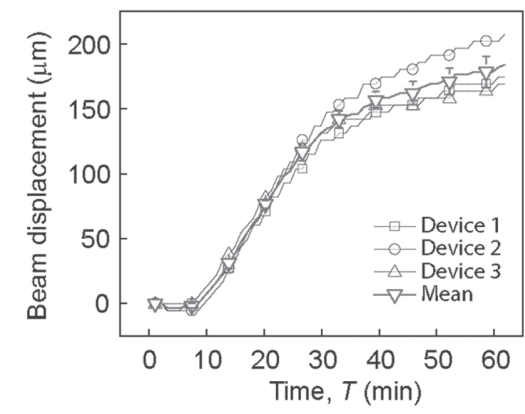

(h)
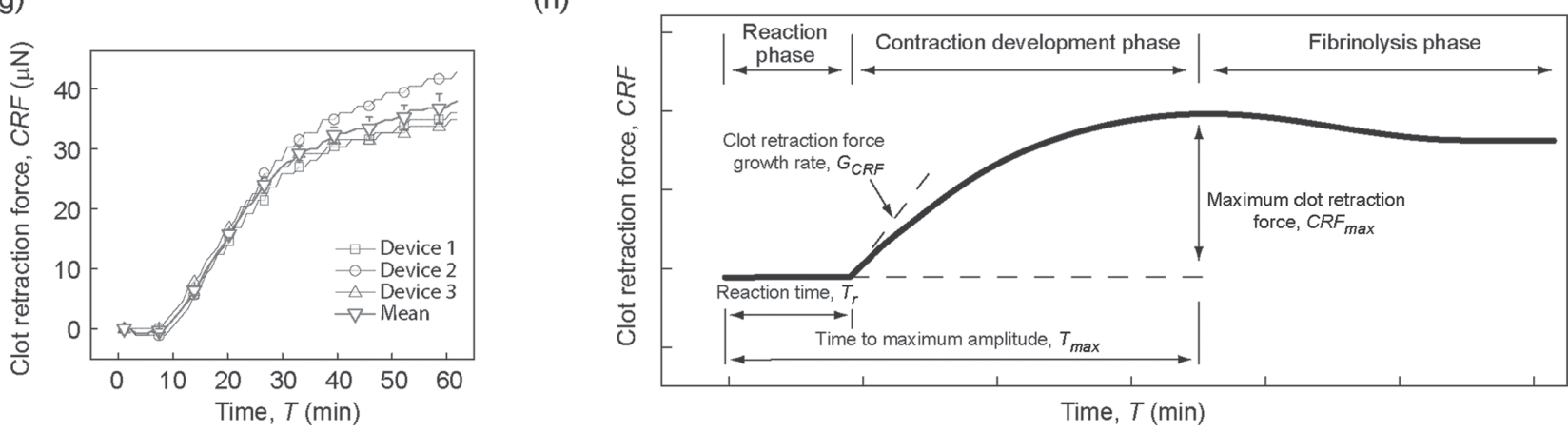

Figure 1. a) Schematic of the mHRM. A structural layer containing two doubly clamped force sensing beams was sandwiched between a top cover layer and a bottom support layer. Each force sensing beam contained a protrusion in the middle of the beam to hold whole blood samples. Positions of fiducial markers engraved onto the protrusions were recorded for quantitative measurements of beam displacements during blood clot formation. b) Photograph of the mHRM device. c) FEM simulation of beam displacement showing stress distribution and beam deformation when a prescribed horizontal load of $200 \mu \mathrm{N}$ was applied uniformly on the beam protrusion. d) Beam displacement as a function of horizontal load. Linear fitting was used to determine the spring constant $K$ of the force sensing beam. e) Representative micrographs showing the dynamic process of blood clot formation at different time points. Blood clotting and contraction caused the two opposite force sensing beams to bend toward each other. Positions of fiducial markers were determined through image processing for quantitative measurements of beam displacements and thus clot retraction force (CRF). Scale bar, $1 \mathrm{~mm}$. $\mathrm{f}$ ) Beam displacement as a function of time, with $t=0$ being the moment of thrombin and calcium addition (see Experimental Section), for three different mHRM devices. Data represent the mean \pm s.e.m. g) Clot retraction force (CRF) calculated based on the spring constant $K$ of the doubly clamped force sensing beam and beam displacement. h) Schematic of dynamic evolution of the CRF during the whole blood coagulation process. Key parameters, including reaction time $T_{\mathrm{r}}$, time to maximum amplitude $T_{\max }$, CRF growth rate $G_{\mathrm{CRF}}$, and maximum $C R F C R F_{\max }$, were highlighted..

of PDMS strain sensing beams and their distance, critical for precise measurements of clot retraction force. The width, length, and thickness of doubly clamped PDMS strain sensing beams used in this work were $1.0,35.5$, and $2.0 \mathrm{~mm}$, respectively. The Young's modulus of PDMS was further calibrated using a standard tensile testing protocol to be about $300 \mathrm{kPa}$ (see Experimental Section), consistent with previously published data. ${ }^{[19]}$ We further conducted FEM simulations to determine the effective spring constant $K$ of the doubly clamped PDMS strain sensing beams $\left(K=0.206 \mu \mathrm{N} \mathrm{m}^{-1}\right)$, which was then used directly for quantification of clot retrac- tion force by multiplying $K$ with beam displacement (Hooke's law; Figure 1c,d). During blood coagulation assays, $12.75 \mu \mathrm{L}$ citrated whole blood was suspended between the two protrusions of PDMS strain sensing beams by pipetting, before another $0.75 \mu \mathrm{L} \mathrm{CaCl} 2$ solution $(0.2 \mathrm{M})$ was added into the blood for re-calcification and initiation of blood clotting. In order to understand if PDMS surface has any intrinsic effect on blood coagulation, studies were undertaken in which we coated TEG cups (blood receptacles) with PDMS. Blood was then added and TEG measurements were performed. Results indicated that when compared to non-PDMS-coated 
controls, PDMS had no measurable effect on blood coagulation (Figure S2, Supporting Information).

Figure 1e demonstrates a temporal series of representative bright-field images showing clotting of a blood drop suspended between the two protrusions of PDMS strain sensing beams recorded using a digital camera immediately after blood re-calcification (designated as $T=0$ ). This blood coagulation assay was conducted at $25^{\circ} \mathrm{C}$, with $1 \mu \mathrm{L}$ thrombin (100 $\mathrm{U} \mathrm{mL}^{-1}$ ) added into the blood prior to $\mathrm{mHRM}$ measurement. Thrombin, as a common clotting factor, is an important intermediate product of the blood coagulation process and cleaves fibrinogen to form fibrin networks. Thus, adding thrombin into blood can expedite the coagulation cascade in a positive feedback fashion. Importantly, thrombin also directly activates platelets and induces platelet contraction. ${ }^{[20]}$ Imaging analysis was conducted to quantify displacement of fiducial markers (and thus strain sensing beams) as a function of time to determine dynamic clot retraction force (CRF) during blood clotting (Figure 1f,g). Data of PDMS strain sensing beam displacement and clot retraction force shown in Figure 1f,g were obtained from three separate coagulation assays using three different mHRM devices, suggesting consistent measurements and satisfactory reproducibility of the mHRM device for clot retraction force measurements (the SEMs are generally around $6 \%$ of the mean CRF).

\subsection{Dynamic Clot Retraction Force Development}

Kinetic curves of clot retraction force obtained by the mHRM device helped reveal different major phases of clot formation (Figure 1h), consistent with those revealed by TEG (Figure S3, Supporting Information). In the first reaction phase between blood re-calcification $(T=0)$ and onset of clot retraction force development $\left(T=T_{\mathrm{r}}\right)$, clot retraction force was essentially zero and not detected by the mHRM (Figure 1f,g). In this reaction phase, the coagulation cascade was underway for formation of the fibrin network needed for clot retraction force development. Onset of clot retraction force development occurred as soon as the fibrin network was in place, and interactions between activated platelets and fibrin strands led to a rapid increase of clot retraction force and initiation of blood clot contraction ("the contraction development phase"; Figure 1h). In the contraction development phase, clot retraction force CRF increased rapidly before approaching a plateau $\left(C R F_{\max }\right.$; Figure $\left.1 \mathrm{~h}\right)$. We designated duration of the reaction phase as $T_{\mathrm{r}}$ and the time it took for clot retraction force to reach its maximum as $T_{\max }$. Growth rate of clot retraction force $G_{\mathrm{CRF}}$ was defined as $G_{\mathrm{CRF}}=\mathrm{d} C R F / \mathrm{d} T @ T=T_{\mathrm{r}}$. Kinetic curves of clot retraction force measured by the mHRM further revealed a clot lysis phase immediately following the contraction development phase, similar to those observed in TEG. In the fibrinolysis phase, clot retraction force decreased slowly from the plateau value $C R F_{\max }$ due to fibrin network being disintegrated. Together, the reaction time $T_{\mathrm{r}}$, time to maximum clot retraction force $T_{\max }$, clot retraction force growth rate $G_{\mathrm{CRF}}$, and maximum clot retraction force $C R F_{\max }$ measured by the mHRM provided a set of parameters for us to assess the full complexity of the coagulation system and for global measurement of the hemostasis under different clotting agent and pro- and anti-coagulant treatments.

\subsection{Effect of Temperature on Clot Tetraction Force}

Low body temperature (hypothermia) is known to slow down blood clotting process. ${ }^{[21]}$ To illustrate the utility of the mHRM for blood coagulation tests, we first examined the effect of temperature on clot retraction force development using the mHRM. Blood coagulation was initiated by re-calcification but without supplementation of the clotting factor thrombin. Compared to that at room temperature $\left(25{ }^{\circ} \mathrm{C}\right)$, development of clot retraction force during blood coagulation was significantly expedited at $37{ }^{\circ} \mathrm{C}$, with significantly shortened reaction time $T_{\mathrm{r}}$ and time to maximum amplitude $T_{\max }$ (Figure 2a). Growth rate of clot retraction force $G_{\mathrm{CRF}}$ was significantly increased at $37{ }^{\circ} \mathrm{C}$ as compared to $25^{\circ} \mathrm{C}$ (Figure 2a). Specifically, the reaction time $T_{\mathrm{r}}$, time to maximum amplitude $T_{\max }$, and growth rate of clot retraction force $G_{\mathrm{CRF}}$ were $8.70 \pm 0.62 \mathrm{~min}$, $22.8 \pm 1.59 \mathrm{~min}$, and $3.50 \pm 0.59 \mu \mathrm{N} \mathrm{m^{-1 }}$, respectively, at $37{ }^{\circ} \mathrm{C}$ (Figure $2 \mathrm{~b}$ ). While at $25{ }^{\circ} \mathrm{C}, T_{\mathrm{r}}, T_{\max }$, and $G_{\mathrm{CRF}}$ were $24.63 \pm 3.10 \mathrm{~min}, 80.25 \pm 4.96 \mathrm{~min}$, and $0.80 \pm 0.15 \mu \mathrm{N} \mathrm{min}{ }^{-1}$, respectively (Figure $2 b$ ). Even though the dynamic properties of clot retraction force development appeared sensitive (a)

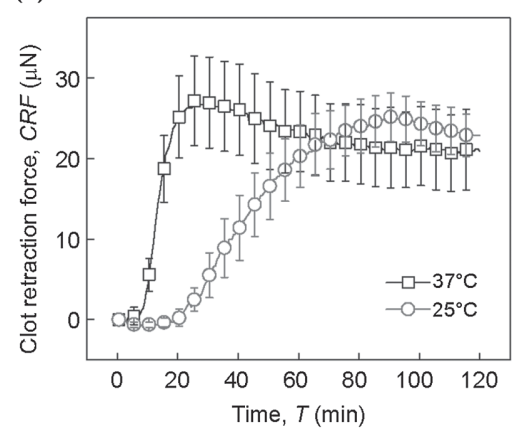

(b)

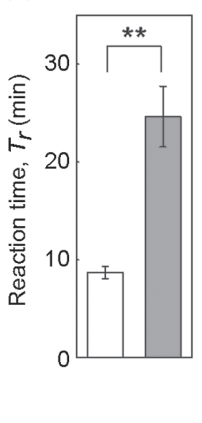

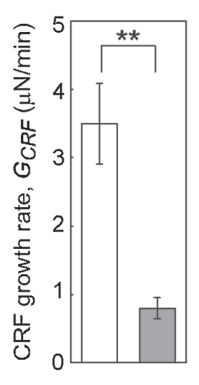

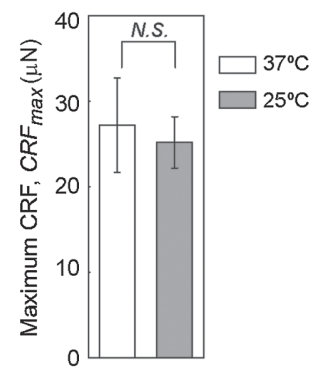

Figure 2. Effect of temperature on clot retraction force. a) Clot retraction force CRF measured by mHRM plotted as a function of time, at both $37{ }^{\circ} \mathrm{C}$ and $25^{\circ} \mathrm{C}$. b) Bar plots of reaction time $T_{\mathrm{r}}$, time to maximum amplitude $T_{\max }$, CRF growth rate $G_{\mathrm{CRF}}$, and the maximum CRF CRF $F_{\max }$ as a function of temperature. Data represent mean $\pm \mathrm{SEM}$ with $n=4$ at $25^{\circ} \mathrm{C}$ and $n=5$ at $37^{\circ} \mathrm{C} .{ }^{* *}, P<0.01$. N.S., $P>0.05$. 
to temperature, the maximum clot retraction force $C R F_{\max }$ was not significantly different between $37^{\circ} \mathrm{C}$ and $25^{\circ} \mathrm{C}$, with $C R F_{\text {max }}=27.21 \pm 5.53 \mu \mathrm{N}$ at $37^{\circ} \mathrm{C}$ and $C R F_{\text {max }}=25.19 \pm$ $3.01 \mu \mathrm{N}$ at $25{ }^{\circ} \mathrm{C}$ (Figure $2 \mathrm{~b}$ ). These temperature-dependent dynamic behaviors were likely the direct effect of different enzyme activity at different temperatures. At $37^{\circ} \mathrm{C}$, coagulation-related enzymes, such as the clotting factors, were more active and thus the coagulation cascade progressed rapidly. In contrast, at $25^{\circ} \mathrm{C}$, these enzymes were less active, so the reaction phase became longer and the CRF also took longer to develop.

\subsection{Effect of Thrombin on Clot Retraction Force}

We conducted blood coagulation assays using the mHRM at $25^{\circ} \mathrm{C}$ to specifically examine the effect of the clotting factor thrombin on clot retraction force development. After blood re-calcification, $1 \mu \mathrm{L}$ thrombin $\left(100 \mathrm{U} \mathrm{mL}^{-1}\right)$ was added into the blood prior to $\mathrm{mHRM}$ measurement. Indeed, compared to re-calcified blood without thrombin treatment, thrombin activation resulted in significantly shortened reaction time $T_{\mathrm{r}}$ before the onset of clot retraction force development and greater growth rate of clot retraction force $G_{\mathrm{CRF}}$ (Figure 3a,b). Specifically, with thrombin treatment, the reaction time $T_{\mathrm{r}}$ and growth rate of clot retraction force $G_{\mathrm{CRF}}$ were $7.50 \pm 0.34 \mathrm{~min}$ and $1.60 \pm 0.08 \mu \mathrm{N} \mathrm{min}{ }^{-1}$, respectively, whereas without thrombin activation, $T_{\mathrm{r}}$ and $G_{\mathrm{CRF}}$ were $24.63 \pm 3.10 \mathrm{~mm}$ and $0.80 \pm 0.15 \mu \mathrm{N} \mathrm{min}{ }^{-1}$, respectively. One hour after blood re-calcification $(T=60 \mathrm{~min})$, the clot retraction force reached a level of $37.12 \pm 2.77 \mu \mathrm{N}$ under thrombin treatment $\left(C R F_{60}=37.12 \pm 2.77 \mu \mathrm{N}\right)$, much greater compared to untreated control $\left(C R F_{60}=25.19 \pm 3.01 \mu \mathrm{N}\right)$.

\subsection{Effects of Pro- and Anticoagulants on Clot Retraction Force}

To demonstrate the effectiveness of the mHRM for rapid assessment of the effects of procoagulant and anticoagulant medications on the global hemostasis response, we conducted comparative studies using the mHRM at $25^{\circ} \mathrm{C}$ on blood samples treated with either aprotinin (procoagulant; $80 \mu \mathrm{g} \mathrm{mL}^{-1}$ ) or heparin (anticoagulant; $8 \mathrm{U} \mathrm{mL}^{-1}$ ). Blood samples were re-calcified and supplemented with thrombin after treatments with aprotinin or heparin. Aprotinin is a broadspectrum serine protease inhibitor with significant abilities to inhibit fibrinolysis. It has been used in coronary artery bypass grafting surgery to reduce blood loss and transfusion requirement. ${ }^{[22]}$ As a well-studied anticoagulant, heparin can bind and potentiate antithrombin III, a major inhibitor of thrombin. Thus, treatment of heparin can effectively inhibit blood coagulation. ${ }^{[23]}$ As expected, heparin treatment indeed suppressed blood clotting completely (Figure 4a). No clot retraction force was detected by the $\mathrm{mHRM}$ during the entire $1 \mathrm{~h}$ assay period. In fact, blood samples treated by heparin were still in a liquid phase with no gelation observed at the end of the assay. We should also note that heparin-treated blood samples, with their clotting completely suppressed, served as negative control demonstrating that the intrinsic viscoelastic property of blood had minimal effect on inducing displacements of force-sensing beams in the mHRM (Figure 4a).

Aprotinin-treated blood, in distinct contrast, demonstrated rapid blood clotting and strong clot retraction. The reaction time $T_{\mathrm{r}}$ and growth rate of clot retraction force $G_{\mathrm{CRF}}$ for aprotinin treated blood were comparable to blood samples treated with thrombin alone (for aprotinin treated blood, $T_{\mathrm{r}}=5.00 \pm 2.08 \mathrm{~min}$, and $G_{\mathrm{CRF}}=1.71 \pm 0.10 \mu \mathrm{N} \mathrm{min}{ }^{-1}$; for thrombin treated control, $T_{\mathrm{r}}=7.50 \pm 0.34 \mathrm{~min}$, and $\left.G_{\mathrm{CRF}}=1.60 \pm 0.08 \mu \mathrm{N} \mathrm{m^{-1 }}\right)$. However, clot retraction force at $T=60 \mathrm{~min}\left(C R F_{60}\right)$ was significantly greater for aprotinin-treated blood compared to thrombin treated control $\left(C R F_{60}=51.38 \pm 2.70 \mu \mathrm{N}\right.$ for aprotinin treated blood, and $C R F_{60}=37.12 \pm 2.77 \mu \mathrm{N}$ for thrombin-treated control, Figure $4 b)$. This observation was consistent with the functional role of aprotinin in inhibiting fibrinolysis and preserving platelet contractile function during blood clotting.

\subsection{Comparison of mHRM with TEG}

Coagulation diagnostics using whole blood samples is currently conducted with technologies such as TEG, ROTEM, (a)

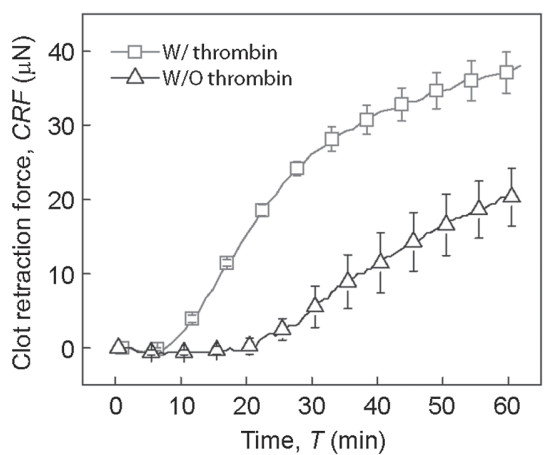

(b)

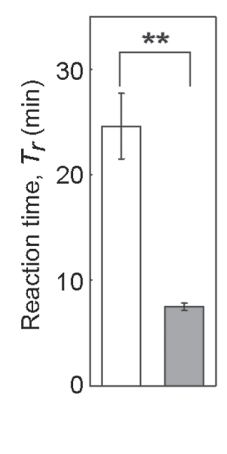

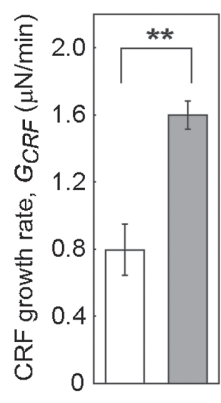

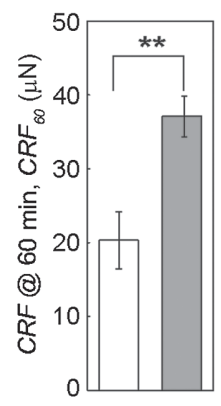

$\square$ W/Othrombin W/thrombin

Figure 3. Effect of thrombin activation on clot retraction force. All experiments were conducted at $25^{\circ} \mathrm{C}$. a) Clot retraction force CRF measured by mHRM plotted as a function of time, for blood samples with or without thrombin treatment, as indicated. b) Bar plots of reaction time $T_{\mathrm{r}}$, CRF growth rate $G_{\mathrm{CRF}}$, and $\mathrm{CRF}$ at $60 \mathrm{~min}\left(C R F_{60}\right)$ as a function of thrombin activation. Data represent mean $\pm \mathrm{SEM}$ with $n=6$ (with thrombin) and $n=4$ (without thrombin). ${ }^{\star *}, P<0.01$. 


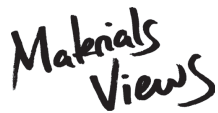

www.MaterialsViews.com

(a)

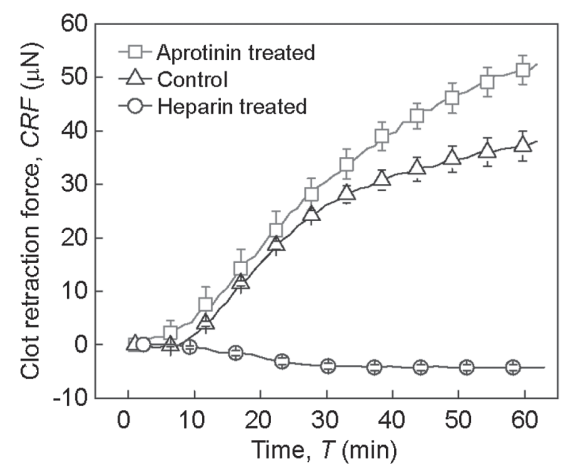

(b)

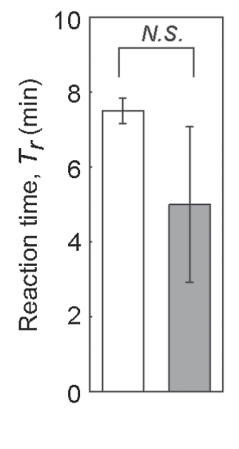

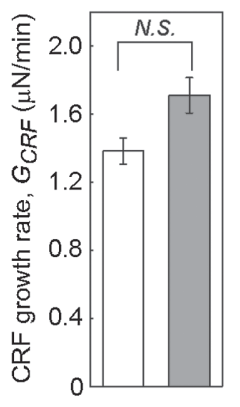

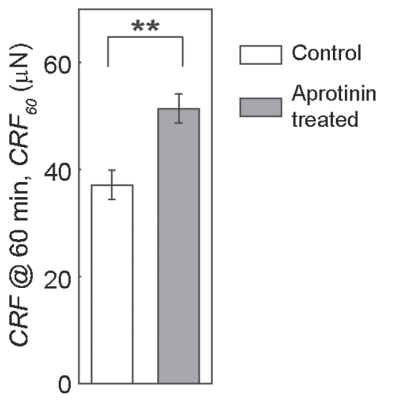

Figure 4. Effect of pro- and anticoagulants on clot retraction force. All experiments were conducted at $25{ }^{\circ} \mathrm{C}$ with blood re-calcified and supplemented with thrombin. a) Clot retraction force CRF measured by mHRM plotted as a function of time, for blood samples pretreated with aprotinin (procoagulant) and heparin (anticoagulant). Control without any pro- or anticoagulant treatment was included for comparison. b) Bar plots of reaction time $T_{\mathrm{r}}$, CRF growth rate $G_{\mathrm{CRF}}$, and $C R F$ at $60 \mathrm{~min}\left(C R F_{60}\right)$. Data represent mean $\pm \mathrm{SEM}$ with $n=3$ (aprotinin treated), $n=6$ (control), and $n=6$ (heparin treated). ${ }^{* \star}, P<0.01$. N.S., $P>0.05$.

and HAS. These whole blood coagulation monitoring methods have provided critical information on the major phases of clot formation and lysis. The HAS system is no longer produced. To compare the kinetic signatures of clot retraction force development measured by mHRM with those obtained from TEG, we conducted additional coagulation assays with recalcified whole blood at both $37^{\circ} \mathrm{C}$ and $25^{\circ} \mathrm{C}$ using TEG. At $37^{\circ} \mathrm{C}$, clot retraction force measured by mHRM showed very similar dynamics with data obtained from TEG (Figure 5a).
The reaction time $T_{\mathrm{r}}$ and time to maximum amplitude $T_{\max }$ obtained from mHRM were $8.70 \pm 0.62 \mathrm{~min}$ and $22.8 \pm 1.59$ min, respectively, comparable with values from TEG, where $T_{\mathrm{r}}=7.68 \pm 1.07 \mathrm{~min}$ and $T_{\max }=24.28 \pm 2.46 \mathrm{~min}$ (Figure 5b). However, at $25{ }^{\circ} \mathrm{C}$, clot retraction force development in the mHRM was significantly slower than blood clotting in TEG (Figure 5c). Specifically, reaction time $T_{\mathrm{r}}$ and time to maximum amplitude $T_{\max }$ in the $\mathrm{mHRM}$ were $24.63 \pm 3.10 \mathrm{~min}$ and $80.25 \pm 4.96 \mathrm{~min}$, respectively, while in TEG, $T_{\mathrm{r}}$, and $T_{\max }$ (a)

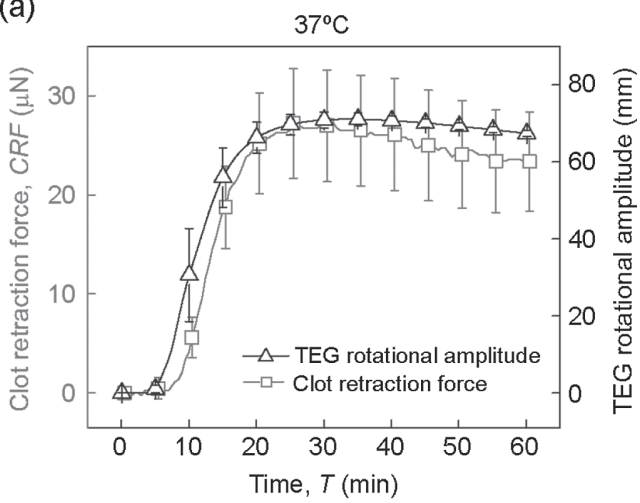

(c)

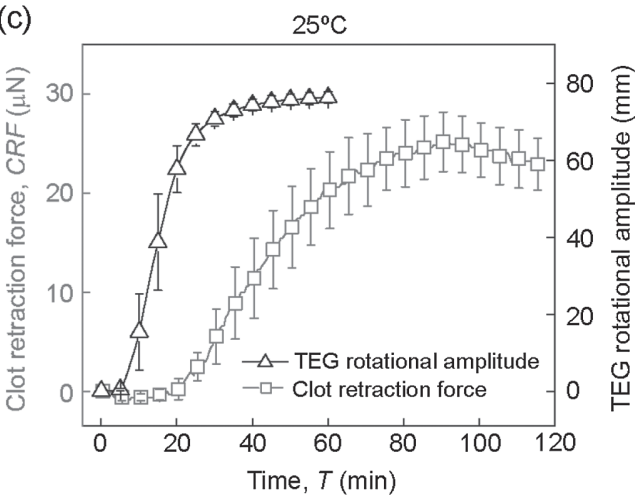

(b)

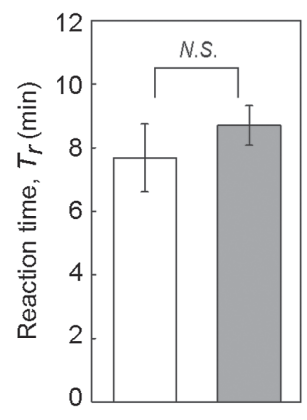

(d)

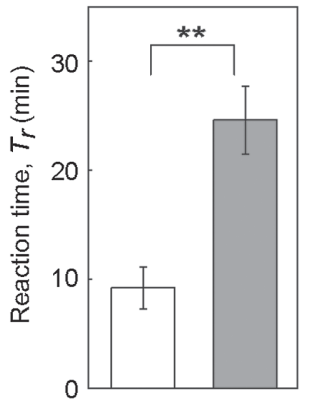

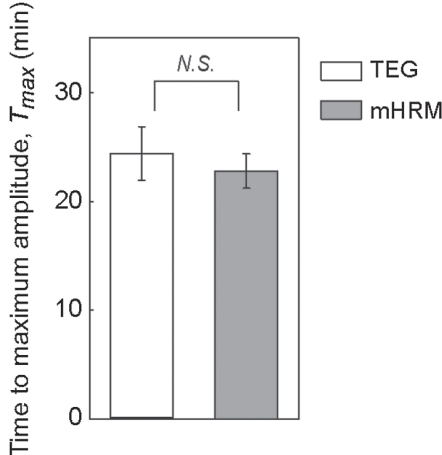

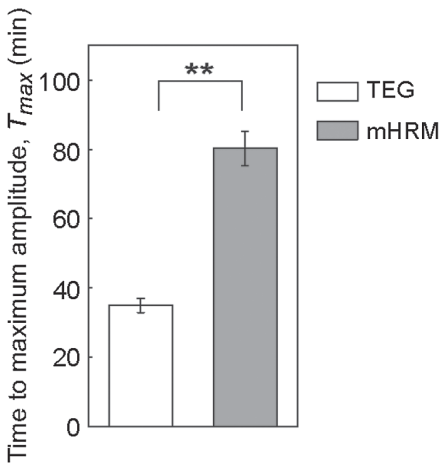

Figure 5. Comparison of mHRM and TEG for blood clotting tests at both a,b) $37^{\circ} \mathrm{C}$ and c,d) $25^{\circ} \mathrm{C}$. a,c) Clot retraction force CRF measured by mHRM and TEG rotational amplitude plotted as a function of assay time, at both a) $37^{\circ} \mathrm{C}$ and c) $25^{\circ} \mathrm{C}$. b,d) Comparison of reaction time $T_{\mathrm{r}}$ (left) and time to maximum amplitude $T_{\max }$ (right) measured by mHRM and TEG at both b) $37^{\circ} \mathrm{C}$ and d) $25^{\circ} \mathrm{C}$, as indicated. Data represent mean \pm SEM with $n=5{ }^{\star \star}, P<0.01$. N.S., $P>0.05$. 
were only $9.22 \pm 1.92 \mathrm{~min}$ and $32.92 \pm 2.07 \mathrm{~min}$, respectively. Slower clot retraction force development in the mHRM at $25{ }^{\circ} \mathrm{C}$ when compared to TEG might be attributed to the shear applied to clotting blood in the TEG. It has been well reported in the literature that fluid shear can increase platelet adhesion, aggregation, and thus activation. Applying quasistatic Couette flow model to TEG experimental setup, the shear rate in TEG was estimated to be about $0.2 \mathrm{~s}^{-1}$, which might be sufficient for eliciting platelet activation. ${ }^{[24]}$ A higher temperature at $37{ }^{\circ} \mathrm{C}$ might lead to expedited biochemical reactions in the coagulation cascade, masking the effect of shear stress on promoting blood coagulation.

\subsection{Future Development for Point-of-Care Testing}

Blood coagulation assays for point-of-care applications will require such assays to be fast, robust, yet easy to operate. In its current form, measurements of clot retraction force by the mHRM take about tens of minutes, which is still suboptimal for point-of-care applications. A potential solution to expedite blood coagulation in the mHRM will be to supplement coagulation activators, such as kaolin, into blood samples, as has been commonly conducted in TEG. Specifically, kaolin can significantly shorten the reaction time $T_{\mathrm{r}}$ and increase the clot retraction force growth rate $G_{\mathrm{CRF}}$ during blood clotting. ${ }^{[25]}$

Currently, the mHRM requires optical microscopy for image collection. Future effort will be devoted to develop a small diagnostic accessory-a "dongle"- that attaches to a smartphone and has the capability for automatic blood sample loading and precise temperature control. Blood coagulation assays can be run automatically by the dongle on a disposable plastic cassette with preloaded reagents, with results captured by smartphone camera and processed simultaneously by the smartphone. We envision that coupling the mHRM technology with recent advances in consumer electronics will lead to the convenience of obtaining quick whole blood coagulation diagnostics results with a single finger prick, making the existing laboratory-based coagulation diagnostics accessible at home and to almost any population with access to smartphones.

Precise metering of blood sample is critical for accurate measurements of blood coagulation properties. In point-ofcare testing using the mHRM, we envision blood sampling to be performed through finger prick in conjunction with a capillary tube that is coupled with a syringe-like dispenser. When blood has been collected in the capillary tube, the dispenser will be connected to the capillary tube and discharge a precise amount of blood onto force sensing elements of the mHRM. Due to hydrophobicity of PDMS and surface tension, blood drops with a prescribed volume will acquire the same shape and position as long as they are properly suspended between force sensing beams in the mHRM.

\section{Conclusion}

The coagulation system is a complex biological system, which balances coagulation and fibrinolysis in an effort to maintain both blood flow and hemostasis. In addition to the acellular (clotting proteins) and cellular (red cells, platelets, white cells) components of the coagulation system, it is now appreciated that coagulation is also affected by inflammation, tissue oxygenation, and endothelial cell function. There is a growing trend in using technologies to examine blood coagulation to monitor standard coagulation therapies in both thrombolic and hemophilic diseases as well as for diagnostic and therapeutic purposes to guide transfusion therapy and treatments for sepsis, traumatic brain injury, and other acute diseases. Coagulation monitoring technologies using whole blood samples such as TEG and ROTEM are now becoming increasingly common because unlike traditional PT and aPTT tests, which examine only the protein portion of the blood, TEG and ROTEM examine both cellular and acellular components involved in blood coagulation and can thus provide a more complete picture of the interactions between the coagulation and inflammatory systems. However, despite the important clinical values that TEG-like technologies can bring, existing whole blood coagulation assays share some critical limitations (such as high cost, limited sample throughput, large footprint, etc.) that have rendered them suboptimal for pointof-care applications. In this research, we have successfully developed a miniaturized clot retraction assay device termed mHemoRetractoMeter (mHRM) using soft elastic material PDMS and replica molding. Doubly clamped flexible PDMS beams were incorporated into the $\mathrm{MHRM}$ design to serve as mechanical strain sensors to report clot retraction force development during blood clotting. Importantly, kinetic curves of clot retraction force obtained by the mHRM have demonstrated different major phases of clot formation (including the reaction phase, contraction development phase, and fibrinolysis phase), consistent with TEG/ROTEM. Key blood clotting parameters including reaction time $T_{\mathrm{r}}$, time to maximum clot retraction force $T_{\max }$, clot retraction force growth rate $G_{\mathrm{CRF}}$, and maximum clot retraction force $C R F_{\max }$ can be conveniently determined by the $\mathrm{mHRM}$, allowing a full assessment of the complexity of the coagulation system and for global measurement of the hemostasis. To demonstrate its clinical utility, the mHRM was utilized for clot retraction force measurements of whole blood samples under different assay temperature, different clotting agent (i.e., thrombin), and pro- (i.e., aprotinin) and anticoagulant (i.e., heparin) treatments. This preliminary effort demonstrated the ability of the mHRM to detect changes in coagulation consistent with those expected when blood was exposed to various proand anticoagulant medications. This lays the foundation to identify novel signatures of blood clotting dynamics under perturbations to different coagulation components, which will allow tailoring of specific coagulation tests and treatments to different clinical applications in the future. The mHRM's low fabrication cost, small size, and consumption of only minute amounts of blood samples make the technology promising as a point-of-care tool for coagulation monitoring.

\section{Experimental Section}

Device Design and Fabrication: The mHRM device consisted of three sandwiched layers, with the middle structural layer 
small

www.MaterialsViews.com

containing two parallel doubly clamped beams as mechanical strain sensors to measure dynamic clot retraction force during blood clotting (Figure 1a). In the mHRM, the structural layer was embedded between a top cover layer and a bottom support layer. Each strain sensing beam contained a protrusion in the middle of the beam to hold a prescribed minute blood sample. In this proofof-concept device, positions of fiducial markers engraved onto the protrusions of the strain sensing beam were recorded using a camera during blood clotting assays for quantitative measurements of beam displacements.

All the three layers of the mHRM device were fabricated through replica molding of soft materials (Figure S1, Supporting Information). Patterns of molds were first generated using AutoCAD (Autodesk, Inc.). A laser cutter was used to cut acrylic sheet (Inventables, Inc.) into desired patterns. These acrylic patterns were affixed to the bottom of a petri dish using double-sided tape before degassed polydimethylsiloxane (PDMS) prepolymer (10:1 base-to-curing-agent ratio) was poured into the petri dish. The dish was placed in a $60{ }^{\circ} \mathrm{C}$ oven overnight, before PDMS was peeled off and cut into desired dimensions to form the top cover and bottom support layers of the mHRM device.

The structure layer of the mHRM device containing doubly clamped strain sensing beams was fabricated using a doublemolding protocol. Briefly, an acrylic pattern was first cut by laser into the same shape as the final structural layer before being used as a mold to generate a negative mold using PDMS (10:1 base-tocuring-agent ratio). Fiducial markers were generated by engraving the acrylic sheet before the sheet was used as a mold. After being cured and peeled off from the acrylic mold, the negative PDMS mold was activated with oxygen/air plasma (PDC-001, Harrick Plasma), followed by surface passivation with trichloro $(1 \mathrm{H}, 1 \mathrm{H}, 2 \mathrm{H}, 2 \mathrm{H}$-perfluorooctyl)silane (Sigma-Aldrich Co.), before degassed PDMS prepolymer (20:1 base-to-curing-agent ratio) was poured onto the mold and excess PDMS was removed using a razor blade. The negative PDMS mold was then placed in a $60^{\circ} \mathrm{C}$ oven for $6 \mathrm{~h}$ before the final structural layer was peeled off from the mold. The structural layer was briefly treated with air plasma before being bonded with the bottom support layer (Figure $1 \mathrm{~b}$ ).

The fabrication protocol was established to generate mHRM devices with consistent geometries (with standard variations less than $4 \%$ of the mean; data not shown). The width, length, and thickness of the doubly clamped PDMS strain sensing beams used in this work were $1.0,35.5$, and $2.0 \mathrm{~mm}$, respectively. The millimeter size scale of the mHRM facilitates its assembly process. Success rate of mHRM fabrication was about $80 \%$, with the cost of a single mHRM device estimated to be about 7 U.S. dollars. We further used an Instron tensile testing machine (Norwood, MA) to calibrate the Young's modulus of PDMS prepared for the structural layer of the mHRM device (Young's modulus, $\approx 300 \mathrm{kPa}$; data not shown).

FEM Simulation: FEM simulation was performed in COMSOL Multiphysics (COMSOL, Inc.). Briefly, a stationary solid mechanics model was developed with a beam subjected to gravity $\left(g=9.8 \mathrm{~m} \mathrm{~s}^{-2}\right)$. A downward vertical force was uniformly exerted on the protrusion surface to mimic the effect of blood weight $(70 \mu \mathrm{N})$. The Young's modulus, density, and Poisson's ratio of PDMS were $300 \mathrm{kPa}, 970 \mathrm{~kg} \mathrm{~m}^{-3}$, and 0.49 , respectively. To simulate the effect of blood clotting and contraction on beam deformation, another horizontal load was applied uniformly on the protrusion
(Figure 1c). Through FEM simulation, horizontal beam displacement was calculated as a function of horizontal load, linear fitting of which provided the effective spring constant $K$ of the PDMS strain sensing beam $\left(K=0.206 \mu \mathrm{N} \mathrm{m}^{-1}\right.$; Figure $\left.1 \mathrm{~d}\right)$, which would be used directly for calculation of clot retraction force in experiments by multiplying $K$ with horizontal beam displacement. The spring constant $K$ of the PDMS strain sensing beam was further validated using the classical beam theory, and $K=0.215 \mu \mathrm{N} \mu \mathrm{m}^{-1}$ ( $K=16 E \delta W^{\beta} / L^{3}$, where $E$ was the Young's modulus, and $\delta, W$, and $L$ were the beam thickness, width, and length, respectively).

Blood Specimen and Coagulation Assays: Under a protocol approved by the University of Michigan's Committee on Care of Use of Animals, whole blood specimens were collected from healthy pigs into a vacutainer (BD Vacutainer, BD Company) containing $3.2 \%$ sodium citrate. All blood samples were assayed within $6 \mathrm{~h}$ after blood collection.

Unless otherwise stated, all clot retraction force measurements were conducted using re-calcified whole blood without additional pretreatment. Briefly, $12.75 \mu \mathrm{L}$ citrated whole blood was suspended between the two protrusions of the PDMS strain sensing beams by pipetting, before another $0.75 \mu \mathrm{L} \mathrm{CaCl}$, solution ( $0.2 \mathrm{~m}$, Fisher Scientific) was added into the blood drop. For mixing, a pipette was inserted into the blood and stirred up gently. The top cover layer was then placed on top of the mHRM device, and image acquisition was started immediately using a microscope coupled with a digital camera (Dino-Lite Handheld Digital Microscope, AnMo Electronics Co.) at 1 or 2 frames per min. To minimize blood evaporation, water drops were placed inside the mHRM device, and the surrounding area of the top cover layer was applied with PDMS prepolymer before being placed on the structural layer of the mHRM device. Coagulation assays at $37^{\circ} \mathrm{C}$ were conducted in a stage-top incubation chamber (PM S1, Zeiss, Inc.). Assays at $25^{\circ} \mathrm{C}$ were performed in a room equipped with a thermostat and air conditioning system.

To activate platelets and thus expedite clot retraction force development, $1 \mu \mathrm{L}$ thrombin (100 $\mathrm{U} \mathrm{mL}^{-1}$, Cayman Chemical Company) was added into the blood as a clotting factor. To assay the effects of pro- and anticoagulants on clot retraction force development, aprotinin (80 $\mathrm{g} \mathrm{mL}^{-1}$; Sigma-Aldrich Co.) and heparin ( $8 \mathrm{U} \mathrm{mL}^{-1}$; Sigma-Aldrich Co.) were further added into the blood in separate experiments. Drug-treated blood samples were incubated at room temperature for $1 \mathrm{~h}$ before loaded into the mHRM device for clot retraction force measurements.

Thromboelastography (TEG) was conducted using the TEG 5000 Thrombelastograph Hemostasis Analyzer System (Haemonetics Corp., Braintree, MA) following standard protocols for citrated whole blood samples.

Image Processing and Data Analysis: Images were processed via template matching using MATLAB (MathWorks, Inc.) to obtain displacement data of doubly clamped PDMS strain sensing beams. Briefly, an area in the first image of the beam containing the fiducial markers was selected as the template, and each of the following images was analyzed to determine the best match of this template, the location of which was extracted for beam displacement analysis. Displacements of doubly clamped strain sensing beams were then converted to clot retraction forces CRF using Hooke's law.

The data of clot retraction force during the contraction development phase was fitted using a quadratic function to obtain 
key coagulation parameters, including reaction time $T_{\mathrm{r}}$, time to maximum amplitude $T_{\text {max }}$, CRF growth rate $G_{\mathrm{CRF}}$, and maximum CRF $C R F_{\text {max }}$. Specifically, $T_{\mathrm{r}}$ was defined as the time when CRF started to increase from the baseline level $(C R F=0 \mu \mathrm{N})$, time to maximum amplitude $T_{\max }$ was the time it took for $C R F$ to reach its maximum value $C R F_{\text {max }}$, and $G_{\text {CRF }}$ was defined as the CRF growth rate at $T_{\mathrm{r}}$ $\left(G_{\mathrm{CRF}}=\mathrm{d}_{\mathrm{CRF}} / \mathrm{d} T @ T=T_{\mathrm{r}}\right)$. Statistical analysis was conducted using Origin (OriginLab Corp.). Two-sample unpaired Student's $t$-test was performed, and only $P$ values less than 0.05 were considered statistically significant.

\section{Supporting Information}

Supporting Information is available from the Wiley Online Library or from the author.

\section{Acknowledgements}

The authors acknowledge financial support from the National Science Foundation (ECCS 1231826, CBET 1263889, and CMMI 1536087), the National Institutes of Health (R01 HL119542), the UM-SITU Collaboration on Biomedical Technologies, the Michigan Center for Integrative Research in Critical Care (M-CIRCC), and the Michigan Translational Research and Commercialization for Life Sciences Program (MTRAC). The authors thank Prof. Jack Hu and Tianyang Han for their help on tensile testing of PDMS samples. The Lurie Nanofabrication Facility at the University of Michigan, a member of the National Nanotechnology Infrastructure Network (NNIN) funded by the National Science Foundation, is acknowledged for support in microfabrication.

[1] N. Key, M. Makris, D. O’Shaughnessy, D. Lillicrap, Practical Hemostasis and Thrombosis, Wiley Online Library, Hoboken, NJ, USA 2009.

[2] K. A. Tanaka, N. S. Key, J. H. Levy, Anesth. Analg. 2009, 108, 1433.

[3] B. P. Nuyttens, T. Thijs, H. Deckmyn, K. Broos, Thromb. Res. 2011, $127, \mathrm{~S} 26$.

[4] a) J. S. Bennett, Ann. N. Y. Acad. Sci. 2001, 936, 340; b) C. J. Jen, L. V. McIntire, Cell Motil. 1982, 2, 445.
[5] J. W. Weisel, Science 2008, 320, 456.

[6] T. Lisman, S. H. Caldwell, A. K. Burroughs, P. G. Northup, M. Senzolo, R. T. Stravitz, A. Tripodi, J. F. Trotter, D.-C. Valla, R. J. Porte, J. Hepatol. 2010, 53, 362.

[7] T. Bombeli, D. Spahn, Br. J. Anaesth. 2004, 93, 275.

[8] P. M. Aggeler, Transfusion 1961, 1, 71.

[9] M. A. McMichael, S. A. Smith, Vet. Clin. Pathol. 2011, 40, 140.

[10] A. Chen, J. Teruya, Clin. Lab. Med. 2009, 29, 391.

[11] M. E. Carr Jr., Cell Biochem. Biophys. 2003, 38, 55.

[12] M. Carr Jr., Clin. Lab. Manag. Rev.: Offic. Publ. Clin. Lab. Manag. Assoc. 1995, 9, 312.

[13] a) A. Krishnaswarmi, M. Carr, R. Jesse, M. Kontos, A. Minisi, J. Ornato, G. Vetrovec, E. Martin, Thromb. Haemostasis 1999, 82, 162; b) P. E. Greilich, J. Carr, S. L. Carr, A. S. Chang, Anesth. Analg. 1995, 80, 459; c) M. Carr, S. Carr, T. Tildon, L. Fisher, E. Martin, J. Thromb. Haemostasis 2003, 1, 243.

[14] a) M. Carr, A. Park, S. Zekert, D. Marques, I. Goldman, M. Sobel, Blood 1993, 82, 603a; b) M. Carr, P. MongeMeberg, K. McCardell, S. Carr, Blood 1996, 88, 79b; c) K. McCardell, S. Carr, M. Carr, J. Invest. Med. 1996, 44, $212 \mathrm{a}$.

[15] a) J. Berthier, K. Brakke, E. Furlani, I. Karampelas, V. Poher, D. Gosselin, M. Cubizolles, P. Pouteau, Sens. Actuators, B 2015, 206, 258; b) H. Li, D. Han, G. Pauletti, A. Steckl, Lab Chip 2014. $14,4035$.

[16] R. M. Judith, J. K. Fisher, R. C. Spero, B. L. Fiser, A. Turner, B. Oberhardt, R. Taylor, M. R. Falvo, R. Superfine, Lab Chip 2015, 15, 1385.

[17] O. Cakmak, E. Ermek, N. Kilinc, S. Bulut, I. Baris, I. Kavakli, G. Yaralioglu, H. Urey, Lab Chip 2015, 15, 113.

[18] X. Li, W. Chen, Z. Li, L. Li, H. Gu, J. Fu, Trends Biotechnol. 2014, $32,586$.

[19] K. Khanafer, A. Duprey, M. Schlicht, R. Berguer, Biomed. Microdevices 2009, 11, 503.

[20] I. Cohen, A. de Vries, Nature 1973, 246, 36

[21] M. J. Rohrer, A. M. Natale, Crit. Care Med. 1992, 20, 1402.

[22] a) W. Van Oeveren, M. Harder, K. Roozendaal, L. Eijsman, C. Wildevuur, J. Thorac. Cardiovasc. Surg. 1990, 99, 788; b) W. Dietrich, M. Spannagl, M. Jochum, P. Wendt, W. Schramm, A. Baranky, F. Sebening, Anesthesiology 1990, 73, 1119.

[23] M. Carr Jr., S. Carr, P. Greilich, Thromb. Haemostasis 1996, 75, 674.

[24] a) S. Jackson, W. Nesbitt, E. Westein, J. Thromb. Haemostasis 2009, 7, 17; b) J. J. Sixma, P. G. Groot, Ann. N. Y. Acad. Sci. 1994, 714, 190; c) W. S. Nesbitt, E. Westein, F. J. Tovar-Lopez, E. Tolouei, A. Mitchell, J. Fu, J. Carberry, A. Fouras, S. P. Jackson, Nat. Med. 2009, 15, 665.

[25] A. Banerjee, S. L. Blois, R. D. Wood, J. Vet. Diagn. Invest. 2011, 23, 1109.

Received: January 27, 2016 Revised: March 16, 2016 Published online: June 1, 2016 